\title{
RESEÑA LIBRO: CONTRIBUCIONES DEL DESARROLLO SOCIAL Y HUMANO A LA SOSTENIBILIDAD
}

\author{
Duván EmILIO Ramírez-OSPINA* \\ luz Elena García-Garcia**
}

El libro tiene la pretensión de ser un aporte a lo que Santos (2012) ha denominado la Epistemología del Sur, contribuyendo a la búsqueda de conocimientos y criterios de validez del conocimiento que otorguen visibilidad y credibilidad a las prácticas cognitivas de los pueblos y los grupos sociales que históricamente han sido excluidos; la epistemología del sur apunta fundamentalmente a prácticas de conocimiento que permitan intensificar la voluntad de transformación social; de esta manera, el texto recoge los resultados de trabajos de investigación desarrollados desde el Centro de Investigaciones en Medio Ambiente y Desarrollo (CIMAD) de la Universidad de Manizales (Colombia), en los cuales se han buscado criterios de validez para los conocimientos que sirven de base para las prácticas de los diversos grupos sociales objeto de investigación; los cuales han sido desconocidos por los propios investigadores sociales colombianos.

Contribuciones del desarrollo social y humano a la sostenibilidad, está conformado por ocho capítulos que van desde el análisis de la perspectiva del pensamiento latinoamericano sobre la sostenibilidad hasta la observación de diversas prácticas sociales para hacer frente a diferentes aspectos tanto de tipo natural como social que afectan o pueden afectar las condiciones de vida de distintos grupos sociales; el texto puede ser consultado en: http://ridum.umanizales.edu.co:8080/xmlui/handle/6789/3365 o http://ridum. umanizales.edu.co:8080/xmlui/handle/6789/3365

En este orden de ideas, se inicia con el trabajo de la Profesora Fridzia Izaguirre Díaz De León de la Universidad Autonoma de Occidente (México) y del Profesor Duván Emilio Ramírez de la Universidad de Manizales (Colombia), quienes a manera de abrebocas de este libro, hacen un abordaje del concepto de sostenibilidad desde una perspectiva latinoamericana; partiendo de la necesidad de construir un pensamiento latinoamericano alrededor del tema, para lo cual, tienen como referente teórico el trabajo de autores de América Latina que han

\footnotetext{
*Universidad de Manizales. Manizales, Colombia. E-mail: merca2@uanizales.edu.co (D) orcid.org/0000-0001-5330-9253 Google Scholar

*Universidad de Manizales. Manizales, Colombia. E-mail: luzeg@umanizales.edu.co

(D) orcid.org/0000-0003-3889-1163 Google Scholar
} 
Reseña - contribuciones del desarrollo social y humano a la sostenibilidad

abordado la temática. Se trata de un trabajo de tipo cualitativo que usa la metodología de análisis de contenidos, para interpretar trabajos previamente escritos, se buscan las principales categorías de análisis sobre sostenibilidad usadas por los autores seleccionados y partir de ellas se establecen relaciones orientadas a llegar a unas reflexiones finales y al planteamiento de posibles temas de investigación futuros. Con este capítulo se busca que los discursos orientadores de las prácticas sociales latinoamericanas alrededor de la sostenibilidad sean producidos en su propio contexto y correspondan a sus procesos histórico-culturales y a las problemáticas que pretenden interpretar (Ramírez e Izaguirre, 2018).

El segundo capítulo, elaborado por la Profesora del CIMAD, Luz Elena García García, plantea algunas tensiones en la Línea de Desarrollo Social y Humano (DSH) de la Maestría, respecto a los enfoques epistemológicos en los planos: teórico, metodológico y práctico sobre el desarrollo; el desarrollo humano y social, y lo ambiental. Las tensiones observadas tienen que ver, por un lado, con los anclajes de pensamiento en la lógica de la justificación, a partir de explicaciones, caracterizaciones y análisis que conciben los problemas objeto de estudio como externos al observador, susceptibles de medición y verificación. Por el otro, las intencionalidades de descubrimiento, transformación y posibilidades de pensar de un modo diferente a lo instituido por el conocimiento científico, a partir de perspectivas interpretativas, críticas y complejas para posibilitar nuevas articulaciones, sentidos valóricos y prácticas. De este ejercicio emergen categorías derivadas de las acciones y las prácticas de los participantes, docentes y autores que sitúan en el centro de la reflexión el pensar-sentir, lo que produce tensiones y tendencias en el desarrollo, el reconocimiento de las necesidades y las capacidades a partir de la concepción de un conocimiento que integra lo humano y lo social.

El tercer capítulo, elaborado por la Profesora de la Universidad de Manizales, Marleny Cardona Acevedo, propone construir referentes desde las capacidades para la reflexión sobre el desarrollo en su dimensión social y humana, que lleva a tejer mundos sostenibles desde las relaciones entre los grupos sociales y de estos con la naturaleza. El texto propone tres dimensiones: capacidades, libertades y buena vida; que son fundamentales en la construcción de ambientes donde el desarrollo social y humano dan sentido al desarrollo como categoría de la modernidad y el planeta como el lugar de la vida. En este trabajo, el desarrollo humano es considerado como un ideal de vida, que se logra a través de la educación, ésta a su vez, potencia capacidades sociales, humanas y ciudadanas. Por eso, en la promoción de las capacidades se construyen formas de actuar que permiten "desarrollo" en los seres humanos para la comprensión de sus experiencias de vida en contexto.

El cuarto capítulo, construido por la Profesora de las Universidades de Manizales y la Salle (Colombia), María Inés Baquero, tiene como propósito analizar distintas perspectivas de la categoría cuidado, desde las aproximaciones socráticas referenciadas por Foucault (1990) como las posturas de Gilligan (1982), Nodding (1992) y Nussbaum (1997), así como los desarrollos 
de Boof (2012). Estas posturas refieren la categoría de cuidado que implica ocuparse de sí, el cuidado como una ética relacional con los demás, hasta la noción de cuidado como un sistema de relaciones con la madre tierra. Por tanto, se trata del paradigma del cuidado, como una incorporación inevitable a los retos y desafíos del desarrollo.

El quinto capítulo, elaborado por el Magister en Medio Ambiente y Desarrollo de la Universidad Nacional, Jorge Andrés Rincón Largo, pretende contribuir a la comprensión de las relaciones complejas entre el desarrollo y el medio ambiente; para ello, busca identificar teorías y posiciones en torno a estas relaciones emergentes que repercuten directamente en la calidad de vida de las comunidades y en la sustentabilidad de los territorios. Lo anterior partiendo de preguntas sobre los presupuestos, sentidos y prácticas ambientales en los ámbitos de la gestión, lo institucional y comunitario, y en el marco del desarrollo social y humano, orientados a la transformación de las condiciones de vida en Colombia, en lo local, regional y nacional, dentro de una pluralidad epistemológica y metodológica, acorde con la naturaleza de los problemas y objetos de estudio de este campo. En este orden de ideas, se plantea y privilegia el diálogo entre disciplinas y saberes en la identificación y construcción de nuevas categorías, y las dinámicas, movimientos, tensiones, contradicciones, y potencialidades sociales y humanas del desarrollo propio de las comunidades.

El sexto capítulo, construido por el director del grupo de investigación Inti Rumi de la Institución Universitaria CESMAG de Pasto y egresado de la Maestría en Desarrollo Sostenible y Medio Ambiente de la Universidad de Manizales, Armando José Quijano Vodniza y la Profesora Luz Elena García García del CIMAD-Universidad de Manizales, profundiza en las estrategias comunitarias que los Quillacingas (comunidad indígena ubicada en el municipio de Pasto) han implementado para adaptarse y mitigar los impactos adversos que genera el cambio climático. Este fenómeno afecta aspectos económicos, sociales, culturales y ambientales, como consecuencia del incremento de las emanaciones de gases de efecto invernadero. Se muestran las alternativas endógenas surgidas de las tecnologías ancestrales que han practicado los Quillacingas a partir de su cosmovisión, para afrontar un problema que es de envergadura mundial. Los hallazgos dejan ver dos categorías emergentes: La microverticalidad y la reciprocidad. La primera se constituye una práctica ancestral, como estrategia de adaptación, y la segunda, basada en la energía y en la conexión del indígena con la Madre Tierra, es una estrategia de mitigación para la reforestación y conservación de los bosques.

El séptimo capítulo, elaborado por los egresados de la Maestría en Desarrollo Sostenible y Medio Ambiente de la Universidad de Manizales: Sonia Del Mar González Bonilla, Bernardo Orobio Riofrío, José Fernando Daza Castro y el Profesor de la Universidad de Manizales, Jaime Alberto Restrepo Soto, hace un ejercicio de recuperación sobre saberes relacionados a eventos climáticos extremos (ECE) contenidos en la memoria de tres (3) comunidades afrodescendientes con asentamiento histórico en el Pacífico colombiano. Para lo cual, se 
Reseña - contribuciones del desarrollo social y humano a la sostenibilidad

trabajó con comunidades que tienen título colectivo obtenido en el marco de la Ley 70 de 1993 (Colombia), cuya unidad territorial ancestral corresponde a cuencas hidrográficas, incluyendo la zona marino-costera, las cuales presentan alta vulnerabilidad a ECE y quienes manifestaron previamente su interés en construir estrategias de gestión de riesgos climáticos. La investigación que dio origen a este capítulo es de tipo histórico- hermenéutico, con un diseño cualitativo y, contempla elementos de la investigación participativa y la investigación narrativa. Los resultados integran tanto los saberes en la memoria sobre los conocimientos, prácticas y creencias relacionados con la ocurrencia, recurrencia y respuestas locales a ECE, así como la identificación de la utilidad de estos saberes para la resiliencia a nuevos ECE, la resistencia a otras amenazas a la integralidad de los territorios y el fortalecimiento de la identidad étnico- territorial.

El octavo capítulo, elaborado por el egresado de la Maestría en Desarrollo Sostenible y Medio Ambiente de la Universidad de Manizales, Jorge Enrique Segura Pinzón, y el Profesor de la Universidad de Manizales Jaime Alberto Restrepo Soto, muestra un análisis de los sentidos y prácticas sociales y ambientales frente a la contaminación que genera la industria siderúrgica en una zona rural en la Vereda Bosígas de Sotaquirá, Boyacá, Colombia. Este producto de un trabajo de grado plantea conexiones con el macroproyecto de investigación de la Línea en Desarrollo Social y humano con relación a los sentidos y prácticas ambientales. Al igual que el macro en el que fue inscrita la investigación, su enfoque fue cualitativa-fenomenológica, basada en un diseño narrativo, lo que permitió indagar historias de vida niños, jóvenes y adultos) para construir los sentidos, las prácticas ambientales, la forma de vida y el impacto que ha tenido la siderúrgica en las condiciones humanas, sociales, agrícolas y culturales de los habitantes. La comunidad atribuyó a la proximidad de la industria, causas y consecuencias de las emisiones atmosféricas por la extracción de hierro, con repercusión en la actividad agrícola, la proyección de la economía local, en la salud, el desarraigo de las nuevas generaciones y la ausencia de una adecuada responsabilidad social empresarial, entre otros (Ramírez y García, 2018).

En general, el presente libro muestra una perspectiva crítica acerca de la sostenibilidad y la resignificación de la noción de lo sostenible como categoría de tiempo que terminó siendo objeto de instrumentalización a partir de la racionalización económica, que busca la compensación de los daños generados por la explotación de los recursos naturales; para concebirse como el reconocimiento de una necesidad de darse cuenta de los desequilibrios ambientales y sociales que genera el desarrollo moderno. ¿Cómo pensar la sosteniblidad que reconozca esta dialéctica entre las posibilidades de equilibración y los desequilibrios de los sistemas societales, institucionales y humanos? En este contexto de problematización emerge la necesidad de restaurar el equilibrio entre el ser, el planeta y el universo.

En un cometido más amplio y profundo que el anterior el concepto, la sustentabilidad se ocupa de la construcción de sentidos que posee la vida en el entramado: naturaleza, ser humano y 
contexto, vinculados a través del cuidado. Esta concepción sitúa en el centro de la reflexión a los sujetos en las sociedades y a las culturas alteradas por los desequilibrios ambientales y sociales, para no sumirse en la impotencia y reconocer sus necesidades, motivaciones, capacidades, intereses y posibilidades. En esta connotación, los tiempos son más flexibles y experienciales en el proceso de creación, imaginación y posibilidad del pensar-ser-actuar lo humano y social en la concreción de propuestas viables para la vida. Preguntas como: ¿Quiénes somos? ¿Hacia dónde vamos? ¿Cómo estamos habitando la tierra? ¿Cuándo hablamos de desarrollo, de qué desarrollo estamos hablando? son provocaciones iniciales de nuestra condición humana, a la vez una apuesta política, un horizonte socio-histórico y un desafío del pensar-sentir.

De esta manera, los caminos de acercamiento al conocimiento de lo humano y social atienden una pluralidad del pensamiento ambiental que se despliega en diferentes marcos epistemelógicos, en los planos teóricos, metodológicos y prácticos, situados por sujetos afectados y comprometidos en los problemas que acontecen en una época. En la especificidad del conocimiento de las realidades, desde un sentir-pensar-intervenir diferente a lo acostumbrado, necesariamente otros textos y nuevos discursos entran en escena en esta producción conjunta; los relatos y las narrativas recuperan las palabras de quienes forjan las culturas, las cosmovisiones, las prácticas cotidianas, las huellas indelebles, y la escucha que fluye de lo hablado y lo no dicho aún.

De lo inédito del trabajo realizado con las comunidades incorporadas a la presente experiencia investigativa emergen categorías necesarias para hacer pertinente el pensar-sentir-intervenir diferente, que contribuyen a la transformación social, humana y ambiental: la configuración de lo social, el reconocimiento de las capacidades, las utopías en concreción, las prácticas ambientales, la microverticalidad, entre otras, que fluyen entre el valle y la montaña, la resistencia y la dinamización de la memoria, el cuidado y el desvelo de sí y del otro. Tres ángulos engloban las categorías expuestas, estos son: la construcción de la realidad, los sujetos, y los despliegues humanos y sociales.

\section{Referencias}

Boof, L. (2012). El cuidado necesario. Madrid, España: Editorial Trotta.

Foucault, M. (1990). Tecnologías del yo y otros textos afines. Barcelona, España: Paidós.

Gilligan, C. (1982). La moral y la teoría. Psicología del desarrollo femenino. Ciudad de México, México: Fondo de Cultura Económica.

Noddings, N. (1992). The challenge to care in schools. New York: Teachers College Press.

Nussbaum M. (1997). El cultivo de la humanidad. Una defensa clásica de la educación liberal. Barcelona, España: Paidós. 
Reseña - contribuciones del desarrollo social y humano a la sostenibilidad

Santos, B. (1998). De la mano de Alicia: lo social y lo político en la postmodernidad. Bogotá, Colombia: Siglo del Hombre Editores.

Ramírez, D. y García, L. (2018). Contribuciones del Desarrollo Social y Humano a la Sostenibilidad. Manizales, Colombia: Fondo Editorial de la Universidad de Manizales.

Ramírez, D. e Izaguirre, F. (2018). La sostenibilidad desde la perspectiva del pensamiento latinoamericano. En D. Ramírez. y L. García. (Eds), Contribuciones del desarrollo social y humano a la sostenibilidad (pp. 5-26). Manizales, Colombia: Fondo Editorial Universidad de Manizales. 